\title{
Phylogenetic Relationships and Diversity within the Pasteurella haemolytica Complex Based on 16S rRNA Sequence Comparison and Outer Membrane Protein and Lipopolysaccharide Analysis
}

\author{
R. L. DAVIES, ${ }^{1 *}$ B. J. PASTER, ${ }^{2}$ AND F. E. DEWHIRST ${ }^{2}$ \\ Division of Infection and Immunity, Institute for Biomedical and Life Sciences, University of Glasgow, Glasgow G12 8QQ, \\ Scotland, ${ }^{1}$ and Department of Molecular Genetics, Forsyth Dental Center, Boston, Massachusetts $02115^{2}$
}

\begin{abstract}
The outer membrane protein (OMP) and lipopolysaccharide (LPS) profiles of 30 untypeable isolates of Pasteurella haemolytica were examined by sodium dodecyl sulfate-polyacrylamide gel electrophoresis and compared with the profiles of typeable isolates. The phylogenetic relationships of 28 isolates representing each of the serotypes of $\boldsymbol{P}$. haemolytica and Pasteurella trehalosi, as well as untypeable isolates of $\boldsymbol{P}$. haemolytica, were determined by comparing 16S rRNA sequences. The analysis of the OMP and LPS profiles of the untypeable isolates revealed five groups, which were designated untypeable groups 1 (UG1) through UG5. The UG1 and UG2 isolates had OMP and LPS profiles identical to the profiles of certain serotype A1 and A2 isolates, respectively. Furthermore, UG1 isolates originating from cattle and sheep could be clearly differentiated on the basis of their OMP profiles. The OMP and LPS profiles of UG3 isolates were similar in appearance to the profiles of serotype A11 isolates, suggesting that these two groups are closely related. The OMP profiles of UG4 and UG5 isolates were unique and different from the OMP profiles of the UG1 through UG3 isolates. A comparison of $16 S$ rRNA sequences revealed that typeable isolates of $P$. haemolytica could be divided into the following three groups: (i) serotype A1, A5 through A9, A12 through A14, and A16 isolates, (ii) serotype A2 isolates, and (iii) serotype A11 isolates. The isolates belonging to the first group all had identical sequences, whereas the sequences of isolates belonging to the second and third groups differed from the sequences of the isolates belonging to the first group at two and four base positions, respectively. The sequence data for the untypeable isolates confirmed the conclusions derived from the OMP and LPS analysis. Isolates belonging to UG1 and UG2 were identical to serotype A1 and A2 isolates, respectively; isolates belonging to UG3 were related to serotype A11 isolates, although there was some sequence heterogeneity within this group; and isolates belonging to UG4 and UG5 were more distantly related to $P$. haemolytica than were isolates belonging to UG1 through UG3 and were clearly members of two different species. As expected, isolates of $P$. trehalosi were even more distantly related to $P$. haemolytica than were the untypeable isolates, but there was significantly more sequence variation among the four serotypes of this species than there was among the serotypes of $P$. haemolytica. The correlation of the OMP and LPS data with the 16S rRNA sequence data suggested that OMP and LPS analyses might be useful for preliminary screening and comparing large numbers of isolates in taxonomic and epidemiological studies of the Pasteurellaceae.
\end{abstract}

Pasteurella haemolytica is a gram-negative bacterium that is responsible for economically important diseases of cattle and sheep $(16,19)$. Traditionally, $P$. haemolytica has been subdivided into two biotypes, biotypes $\mathrm{A}$ and $\mathrm{T}$, on the basis of biochemical characteristics, and 17 serotypes on the basis of variations in capsular polysaccharides $(2,35)$. However, biotypes $\mathrm{A}$ and $\mathrm{T}$ differ not only biochemically, but also serologically since serotypes A1, A2, A5 through A9, A11 through $\mathrm{A} 14, \mathrm{~A} 16$, and $\mathrm{A} 17$ are associated only with biotype $\mathrm{A}$, whereas serotypes T3, T4, T10, and T15 are associated only with biotype $T$. In addition to biochemical and serological differences, epidemiological and pathogenic differences between the $\mathrm{A}$ and $\mathrm{T}$ biotypes have long suggested that they are, in fact, different species. Biotype A strains are associated with pneumonic pasteurellosis of cattle and sheep and septicemia in young lambs, whereas biotype $T$ strains cause a well-defined systemic disease of young adult sheep $(16,19)$. The results of studies of Biberstein and Francis (4) and Sneath and Stevens (31) supported

${ }^{*}$ Corresponding author. Mailing address: Division of Infection and Immunity, IBLS, Joseph Black Building, University of Glasgow, Glasgow G12 8QQ, Scotland, United Kingdom. Phone: 0141339 8855, ext. 6685. Fax: 01413304600 . the hypothesis that biotypes $\mathrm{A}$ and $\mathrm{T}$ are different species. In 1990, Sneath and Stevens (32) proposed that biotype T strains of $P$. haemolytica should be recognized as members of a new species, Pasteurella trehalosi. More recently, Dewhirst et al. (13), using 16S rRNA sequence comparisons, demonstrated that $P$. haemolytica and $P$. trehalosi belong to different clusters in the family Pasteurellaceae and may, in fact, belong to different genera. However, it should be noted that both $P$. haemolytica and $P$. trehalosi were excluded from the genus Pasteurella sensu stricto by Mutters et al. (26), although they have not been assigned to a new genus or new genera yet.

In addition to the isolates which belong to one of the recognized serotypes of $P$. haemolytica, a significant number of isolates are untypeable. Fraser et al. (17) reported that 6 to $11 \%$ of ovine isolates were untypeable, and the corresponding value for bovine isolates is higher (29). Nine serogroups of bovine and ovine untypeable $P$. haemolytica isolates were described on the basis of the results of countercurrent immunoelectrophoresis (14). Biberstein (3) suggested that untypeable isolates probably represented unencapsulated mutants belonging to biotype A, although Aarsleff et al. (1) noted that the sugar fermentation patterns of many untypeable isolates differed from those of classical biotype $A$ isolates. On the basis of 
differences in biochemical characteristics, 12 biogroups have been described within the $P$. haemolytica complex $(6,7,25,27)$, and some of these biogroups represent new species $(25,27)$. Although some untypeable isolates belong to the same biogroup as biotype $\mathrm{A}$ isolates of $P$. haemolytica (i.e., biogroup 1), others belong to biogroups 5 through $12(6,7)$. Heterogeneous biogroup 3, which includes serotype A11 isolates, and biogroups 5 and 9 (in particular) are thought to represent new species (25).

From the observations described above it is clear that $P$. haemolytica comprises a diverse range of bacteria and is best described as a complex rather than a single species (27). This situation is undesirable because it makes the results of comparative studies of different isolates of $P$. haemolytica (for example, biotype $\mathrm{A}$ and $\mathrm{T}$ and untypeable isolates) difficult to interpret and, furthermore, may actually hinder studies on the pathogenesis and epidemiology of $P$. haemolytica. In an attempt to ameliorate this situation, the taxonomic relationships of isolates belonging to the $P$. haemolytica complex are currently being examined as part of a wider study of the diversity of this group of organisms. The intraspecific diversity of $P$. trehalosi and $P$. haemolytica has been assessed previously by analyzing variations in the lipopolysaccharide (LPS) and outer membrane protein (OMP) profiles between and within capsular polysaccharide serotypes $(10,12)$. The LPS and OMP profiles of $P$. trehalosi isolates and $P$. haemolytica isolates were shown to be significantly different (12), confirming that these taxa should be recognized as distinct species (32). In the present study, the analysis of the LPS and OMP profiles of isolates belonging to the $P$. haemolytica complex was extended by examining untypeable isolates. In addition, the phylogenetic relationships of isolates belonging to the $P$. haemolytica complex were examined by comparing $16 \mathrm{~S}$ rRNA sequences. The diversity within the $P$. haemolytica complex, including $P$. trehalosi, based on the results of an analysis of the OMP and LPS profiles of 274 isolates could then be compared with the phylogenetic data derived from the 16S rRNA sequences.

\section{MATERIALS AND METHODS}

Bacterial strains and growth conditions. A total of 30 untypcable isolates of $P$. haemolytica, which were obtained from the Moredun Research Institute and British Veterinary Investigation Centres, were compared by analyzing their OMP and LPS profiles. Details concerning these isolates are shown in Table 1. A total of 28 isolates, including field isolates and isolates obtained from the National Collection of Type Cultures (NCTC), which represented each serotype of $P$. haemolytica (except recently described serotype A17 [35]) and $P$. trehalosi, as well as representative isolates belonging to each of the untypeable groups (UG) identified by the OMP and LPS analysis, were subjected to a 16S rRNA sequence comparison analysis. In addition, sequences from seven related reference strains were included for comparison. Details concerning these isolates are shown in Table 2.

Isolates from the Moredun Research Institute and Veterinary Investigation Centres were submitted to the Glasgow laboratory on blood agar slopes. Cultures from the NCTC were obtained in a freeze-dried state and were reconstituted on sheep blood agar (brain heart infusion agar [Oxoid] containing 5\% [vol/vol] defibrinated sheep blood). At the Veterinary Investigation Centres isolates were identified as $P$. haemolytica on the basis of clinical diagnosis, colony morphology, Gram staining, cell morphology, and the results of a preliminary biochemical characterization. Isolates were submitted to the Moredun Rescarch Institute for serotyping $(10,12)$, and those found to be untypeable were included in this study. The isolates were stored at $-70^{\circ} \mathrm{C}$ in $50 \%$ (vol/vol) glycerol in brain heart infusion broth (Oxoid). Subsequently, the bacteria were streaked onto sheep blood agar and grown overnight at $37^{\circ} \mathrm{C}$. For preparation of outer membranes, bacteria were grown for 6 to $7 \mathrm{~h}$ in $500-\mathrm{ml}$ volumes of brain heart infusion broth in 2-liter Erlenmeyer flasks at $37^{\circ} \mathrm{C}$ with shaking at $120 \mathrm{rpm}$.

Preparation of OMPs and LPS. OMPs were obtained by Sarkosyl extraction (15), as described previously (11). LPS was obtained by proteinase K digestion (20) of outer membranes as described previously (9), except that the $2 \times$ sample buffer consisted of $0.125 \mathrm{M}$ Tris- $\mathrm{HCl}(\mathrm{pH} 6.8), 20 \%$ (vol/vol) glycerol, $4 \%$ (wt/vol) sodium dodecyl sulfate (SDS), $10 \%$ (vol/vol) 2-mercaptoethanol, and $0.0025 \%$ (wt/vol) bromophenol blue.
TABLE 1. Details of the untypeable isolates examined in this study

\begin{tabular}{|c|c|c|c|c|c|c|c|}
\hline $\begin{array}{l}\text { Laboratory } \\
\text { designation }\end{array}$ & $\begin{array}{c}\text { Previous } \\
\text { designation }^{a}\end{array}$ & Group & $\begin{array}{l}\text { LPS } \\
\text { type }\end{array}$ & $\begin{array}{c}\text { OMP } \\
\text { type }\end{array}$ & $\begin{array}{c}\text { Host } \\
\text { species }^{b}\end{array}$ & $\begin{array}{l}\text { Disease } \\
\text { status }^{c}\end{array}$ & $\begin{array}{l}\text { Site of } \\
\text { origin }\end{array}$ \\
\hline PH154 & UT27 & UG1 & $1 \mathrm{~A}$ & 1.1.1 & B & $\mathrm{P}$ & Lung \\
\hline PH156 & UT32 & UG1 & $1 \mathrm{~A}$ & 1.1.1 & B & $P$ & Lung \\
\hline PH318 & UT3 & UG1 & $1 \mathrm{~A}$ & 1.1 .1 & B & $\mathrm{P}$ & Lung \\
\hline PH612 & & UG1 & $1 \mathrm{~A}$ & 1.1.1 & B & $P$ & Lung \\
\hline PH320 & UT6 & UG1 & $1 \mathrm{~A}$ & 1.2 .1 & $\mathrm{O}$ & $\mathrm{P}$ & Lung \\
\hline PH452 & & UG1 & $1 \mathrm{~A}$ & 1.2 .1 & $\mathrm{O}$ & S & Lung \\
\hline PH690 & & UG2 & 3B & 2.2 .2 & $\mathrm{O}$ & $S$ & Liver \\
\hline PH692 & & UG2 & $3 B$ & 2.2 .2 & $\mathrm{O}$ & $\mathbf{P}$ & Lung \\
\hline PH150 & UT18 & UG3 & $4 \mathrm{~A} / \mathrm{C}$ & 3.2 .2 & $\mathrm{O}$ & $\mathrm{P}$ & Lung \\
\hline PH152 & UT23 & UG3 & $4 \mathrm{~A} / \mathrm{C}$ & 3.2 .2 & B & $\mathrm{P}$ & Lung \\
\hline PH290 & & UG3 & $4 \mathrm{~A} / \mathrm{C}$ & 3.2 .2 & $\mathrm{O}$ & $\mathrm{Ab}$ & Stomach \\
\hline PH474 & & UG3 & $4 \mathrm{~A} / \mathrm{C}$ & 3.2 .2 & $\mathrm{O}$ & $\mathrm{OL}$ & Gingiva \\
\hline PH496 & & UG3 & $4 \mathrm{~A} / \mathrm{C}$ & 3.2 .2 & $\mathrm{O}$ & $\mathrm{H}$ & Lung \\
\hline PH574 & & UG3 & $4 \mathrm{~A} / \mathrm{C}$ & 3.2 .2 & $\mathrm{O}$ & & Gut \\
\hline PH682 & & UG3 & $4 \mathrm{~A} / \mathrm{C}$ & 3.2 .2 & $\mathrm{O}$ & $\mathrm{P}$ & Lung \\
\hline PH700 & & UG3 & $4 \mathrm{~A} / \mathrm{C}$ & 3.2 .2 & $\mathrm{O}$ & $\mathrm{H}$ & Semen \\
\hline PH774 & & UG3 & $4 \mathrm{~A} / \mathrm{C}$ & 3.2 .3 & $\mathrm{O}$ & S & $\begin{array}{l}\text { Lymph } \\
\text { node }\end{array}$ \\
\hline PH40 & UT35 & UG4 & & 4.1 .1 & B & $\mathrm{P}$ & Lung \\
\hline PH144 & UT2 & UG4 & & 4.1.1 & B & $\mathbf{P}$ & Lung \\
\hline $\mathrm{PH} 282$ & & UG4 & & 4.1.1 & B & $\mathrm{P}$ & Lung \\
\hline PH378 & & UG4 & & 4.1.1 & B & $\mathbf{P}$ & Lung \\
\hline PH686 & & UG4 & & 4.1 .1 & B & $\mathbf{P}$ & Lung \\
\hline PH810 & & UG4 & & 4.1 .1 & B & $\mathrm{P}$ & Lung \\
\hline PH354 & & UG4 & & 4.1 .1 & $\mathrm{O}$ & & \\
\hline PH808 & & UG4 & & 4.1 .1 & B & $\mathrm{Ab}$ & Placenta \\
\hline PH360 & & UG4 & & 4.1.2 & B & & Liver \\
\hline PH652 & & UG4 & & 4.1.2 & B & $\mathrm{Ab}$ & Placenta \\
\hline PH670 & & UG4 & & 4.1 .2 & B & G & Flank \\
\hline PH704 & & UG5 & & 5.1 .1 & B & $\mathrm{Ab}$ & Fetus \\
\hline PH806 & & UG5 & & 5.1 .1 & B & $\mathrm{Ab}$ & Placenta \\
\hline
\end{tabular}

${ }^{a}$ See reference 14

${ }^{b} \mathrm{~B}$, bovine; $\mathrm{O}$, ovine.

${ }^{c} \mathrm{P}$, pneumonia; $\mathrm{S}$, septicemia; $\mathrm{Ab}$, abortion; $\mathrm{OL}$, oral lesion; $\mathrm{H}$, healthy; $\mathrm{G}$, granuloma.

Protein assay. The protein concentrations of outer membrane samples were determined by the modified Lowry procedure described by Markwell et al. (24).

SDS-PAGE. OMPs and LPS were separated by SDS-polyacrylamide gel electrophoresis (PAGE) by using the SDS discontinuous system of Laemmli (23), as described previously (11). OMPs were separated in $12 \%(\mathrm{wt} / \mathrm{vol})$ polyacrylamide resolving gels, whereas LPS was separated in $15 \%$ (wt/vol) polyacrylamide resolving gels containing $4 \mathrm{M}$ urea. The protein molecular mass standards (Pharmacia) used were phosphorylase b $(94 \mathrm{kDa})$, bovine serum albumin $(67 \mathrm{kDa})$, ovalbumin (43 kDa), carbonic anhydrase $(30 \mathrm{kDa})$, trypsin inhibitor $(20.1 \mathrm{kDa})$, and $\alpha$-lactalbumin $(14.4 \mathrm{kDa})$. OMPs were visualized by staining the gels with Coomassic brilliant blue, and LPS was visualized by silver staining (34). The molecular masses of individual proteins were estimated from calibration curves.

Crude DNA isolation. Bacteria were grown overnight in 5-ml volumes of brain heart infusion broth. A $1-\mathrm{ml}$ portion of each culture was transferred to an Eppendorf tube and centrifuged at $15,000 \times g$ for $1 \mathrm{~min}$. The supernatant was removed, and the cells were resuspended in $200 \mu$ l of resuspension buffer $(10$ $\mathrm{mM}$ Tris-HCl [pH 7.6], $1 \mathrm{mM}$ EDTA). DNA was released by using GeneReleaser (Bioventures, Inc.) according to the manufacturer's instructions. A $15-\mu 1$ portion of GeneReleaser was mixed with $5 \mu$ l of the cell suspension, a bead of Ampliwax (PCR Gem 100) was added, and the tube was microwaved on high for $5 \mathrm{~min}$.

Amplification of 16S rRNA cistrons. The 16S rRNA cistrons were amplified with primers 1 and 2 (Table 3). PCRs were performed in thin-walled tubes with a Perkin-Elmer model 480 thermal cycler. The PCR was carried out with a Perkin Elmer Gene Amp PCR kit by using a hot-start protocol as suggested by the manufacturer. The following conditions were used for amplification: denaturation at $94^{\circ} \mathrm{C}$ for $45 \mathrm{~s}$, annealing at $50^{\circ} \mathrm{C}$ for $45 \mathrm{~s}$, and elongation at $72^{\circ} \mathrm{C}$ for $45 \mathrm{~s}$, with an additional $5 \mathrm{~s}$ added for each cycle. A total of 30 cycles were performed, and this was followed by a final elongation step at $72^{\circ} \mathrm{C}$ for $15 \mathrm{~min}$. 
TABLE 2. Details of the isolates used in the $16 \mathrm{~S}$ rRNA sequence comparison

\begin{tabular}{|c|c|c|c|c|c|}
\hline Taxon & Laboratory designation(s) & Previous designation(s) & $\begin{array}{l}\text { Serotype or } \\
\text { group }\end{array}$ & Host species ${ }^{a}$ & $\begin{array}{c}\text { GenBank } \\
\text { accession no. }\end{array}$ \\
\hline P. haemolytica & PH2, PH8 & None, none & A1 & $\mathrm{B}, \mathrm{O}$ & U57066 \\
\hline P. haemolytica & PH160 $0^{\mathrm{T}}, \mathrm{PH} 208, \mathrm{PH} 380^{b}$ & NCTC $9380^{\mathrm{T}}$, none, none & $\mathrm{A} 2$ & $\mathrm{O}, \mathrm{B}, \mathrm{O}$ & M75080 \\
\hline P. haemolytica & PH568 & None & A5 & $\mathrm{O}$ & U57066 \\
\hline P. haemolytica & PH284, PH376 & None, none & A6 & $\mathrm{O}, \mathrm{B}$ & U57066 \\
\hline P. haemolytica & PH296, PH388 & None, none & A7 & $\mathrm{O}, \mathrm{O}$ & U57066 \\
\hline P. haemolytica & PH236 & NCTC 10637 & A8 & $\mathrm{O}$ & U57066 \\
\hline P. haemolytica & PH374 & None & A9 & $\mathrm{O}$ & U57066 \\
\hline P. haemolytica & PH344 & None & A11 & $\mathrm{O}$ & U57067 \\
\hline P. haemolytica & PH346 & None & $\mathrm{A} 12$ & $\mathrm{O}$ & U57066 \\
\hline P. haemolytica & PH570 & None & A13 & $\mathrm{O}$ & U57066 \\
\hline P. haemolytica & PH66 & NCTC 11303 & A14 & $\mathrm{O}$ & U57066 \\
\hline P. haemolytica & PH706 & None & A16 & $\mathrm{O}$ & U57066 \\
\hline P. haemolytica & PH318 & UT3 & UG1 & B & U57066 \\
\hline P. haemolytica & PH690 & None & UG2 & $\mathrm{O}$ & M75080 \\
\hline P. haemolytica & PH152 & UT23 & UG3 & B & U57068 \\
\hline P. haemolytica & PH290 & None & UG3 & $\mathrm{O}$ & U57069 \\
\hline P. haemolytica & PH40 & UT35 & UG4 & B & U57070 \\
\hline P. haemolytica & PH 360 & None & UG4 & $\mathbf{B}$ & U57071 \\
\hline P. haemolytica & PH704 & None & UG5 & B & U57072 \\
\hline P. trehalosi & PH68 & NCTC 11550 & T3 & $\mathrm{O}$ & U57073 \\
\hline P. trehalosi & PH252 & NCTC 10641 & $\mathrm{~T} 10$ & $\mathrm{O}$ & U57074 \\
\hline P. trehalosi & $\mathrm{PH} 70$ & NCTC 10624 & $\mathrm{~T} 15$ & $\mathrm{O}$ & M75063 \\
\hline P. trehalosi & PH246 & NCTC 10626 & $\mathrm{~T} 4$ & $\mathrm{O}$ & U57075 \\
\hline Bisgaard's taxon 15 & & CCUG 16500 & & $\mathrm{P}$ & U57076 \\
\hline Bisgaard's taxon 18 & & CCUG 20954 & & $\mathrm{O}$ & U57077 \\
\hline Bisgaard's taxon 20 & & CCUG 16504 & & $\mathbf{R}$ & U57078 \\
\hline A. capsulatus & & NCTC $11408^{\mathrm{T}}$ & & $\mathbf{R}$ & M75062 \\
\hline H. paracuniculus & & ATCC $29986^{\mathrm{T}}$ & & $\mathbf{R}$ & M75077 \\
\hline H. parasuis & & Nagasaki & & $\mathbf{P}$ & M75066 \\
\hline H. parasuis & & NCTC $4557^{\mathrm{T}}$ & & $\mathbf{P}$ & M75065 \\
\hline
\end{tabular}

${ }^{a} \mathrm{~B}$, bovine; $\mathrm{O}$, ovine; $\mathrm{P}$, porcine; $\mathrm{R}$, rabbit.

${ }^{b} \mathrm{~T}=$ type strain.

The purity of the amplified product was determined by electrophoresis in a $1 \%$ agarose gel (FMC Bioproducts). DNA was stained with ethidium bromide and viewed under short-wavelength UV light.

Purification of PCR products. The amplified DNA was purified by precipitation with polyethylene glycol $8000(22)$. The DNA mixture was transferred to a fresh tube, 0.6 volume of $20 \%$ polyethylene glycol 8000 (Sigma) in $2.5 \mathrm{M} \mathrm{NaCl}$ was added, and the mixture was incubated at $37^{\circ} \mathrm{C}$ for $10 \mathrm{~min}$. The sample was centrifuged for $30 \mathrm{~min}$ at $15,000 \times \mathrm{g}$, and the resulting pellet was washed with ice-cold $80 \%$ ethanol. This preparation was centrifuged as described above, and the resulting pellet was air dried, dissolved in $30 \mu \mathrm{l}$ of distilled water, and used for cycle sequencing as described below.

Sequencing methods. The DNA sample obtained from the PCR was directly sequenced with a cycle sequencing kit (Promega fmol DNA sequencing system) by following the manufacturer's protocol. Details concerning the six sequencing primers used (primers 3 through 8 ) are shown in Table 3 . The primers were end labelled with ${ }^{33} \mathrm{P}$ (Dupont, NEN) by using the protocol recommended by the manufacturer. Approximately $100 \mathrm{ng}$ of purified DNA from each PCR was used for sequencing. The reaction products were loaded onto $8 \%$ polyacrylamideurea gels and electrophoresed, and bands were detected by exposure to X-ray film for $24 \mathrm{~h}$

16S rRNA data analysis. A program set for data entry, editing, sequence alignment, secondary-structure comparison, similarity matrix generation, and dendrogram construction for 16S rRNA data was written in Microsoft QuickBASIC for use with IBM PC-AT and compatible computers (28). The RNA sequences were entered and aligned as described previously (28). The sequence database contains approximately $10016 \mathrm{~S}$ rRNA sequences of members of the Pasteurellaceae. Similarity matrices were constructed from the aligned sequences by using only those sequence positions for which $90 \%$ of the strains had data. The similarity matrices were corrected for multiple base changes at single positions by the method of Jukes and Cantor (21). Phylogenetic trees were constructed by the neighbor-joining method of Saitou and Nei $(30,33)$.

GenBank accession numbers. The GenBank accession numbers for the strains examined in this study are given in Table 2 .

TABLE 3. Oligonucleotide primers used for PCR amplification and sequencing of $16 \mathrm{~S}$ genes coding for rRNA

\begin{tabular}{cclll}
\hline Primer no. & Designation & \multicolumn{1}{c}{ Type } & \multicolumn{1}{c}{ Sequence $\left(5^{\prime} \text { to } 3^{\prime}\right)^{a}$} & Position $^{b}$ \\
\hline 1 & C70 & PCR & AGAGTTTGATYMTGGC & $8-23$ \\
2 & B37 & PCR & TACGGYTACCTTGTTACGA & $1495-1513$ \\
3 & X12 & Sequencing & ACCAACTACCTAATCCCACTTGGG & $233-256$ \\
4 & B35 & Sequencing & GTRTTACCGCGGCTGCTG & $519-536$ \\
5 & B36 & Sequencing & GGACTACCAGGGTATCTA & $789-806$ \\
6 & C01 & Sequencing & GGTTGCGCTCGTTGCGGG & Reverse \\
7 & X10 & Sequencing & ACGGGCGGTGTGTRC & Reverse \\
8 & C31 & Sequencing & GGAATCGCTAGTAATCG & Reverse \\
& & &
\end{tabular}

\footnotetext{
${ }^{a}$ The base designations are standard International Union of Biochemistry designations for bases and ambiguity.

${ }^{b}$ Position numbers based on $E$. coli positions.
} 


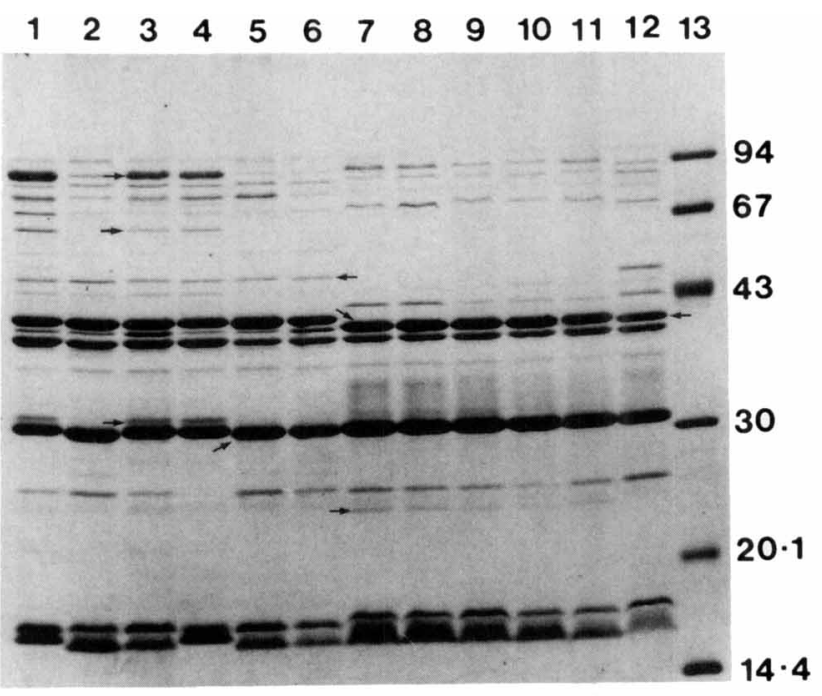

FIG. 1. OMP profiles of untypeable isolates of $P$. haemolytica showing OMP types 1.1.1 (lanes 3 and 4), 1.2.1 (lanes 5 and 6), 3.2.2 (lanes 9 through 11), and 3.2.3 (lane 12). The OMP profiles of serotype A1 isolates that are OMP type 1.1.1 and 1.2.1 isolates are shown in lanes 1 and 2, respectively; the OMP profiles of serotype A11 isolates that are OMP type 3.2.2 isolates are shown in lanes 7 and 8 . Lane 13 contained molecular mass standards, whose positions (in kilodaltons) are indicated on the right.

OMP and LPS profiles of untypeable isolates. The OMP and LPS profiles of 30 untypeable isolates of $P$. haemolytica were compared with the profiles of members of the $12 P$. haemolytica serotypes described previously (10). The results are summarized in Table 1 . On the basis of variations in their OMP profiles, the untypeable isolates could be divided into five major groups, which were designated UG1 through UG5. UG1 consisted of six isolates that had profiles identical to the profiles characteristic of certain serotype A1 isolates (10) and could be divided into two subgroups. Four isolates had type 1.1.1 OMP profiles (Fig. 1, lanes 3 and 4), and two isolates had type 1.2.1 OMP profiles (Fig. 1, lanes 5 and 6). These profiles were identical to the corresponding profiles of serotype A1 isolates, which are shown for comparison in Fig. 1, lanes 1 and 2 . As was the case with the serotype A1 isolates (10), the type 1.1.1 profiles were characterized by the presence of a $31-\mathrm{kDa}$ major protein and 31.5- and 65-kDa minor proteins and enhanced expression of a 90-kDa protein (Fig. 1, lane 3, arrows); the type 1.2.1 profiles were characterized by the presence of a $30.5-\mathrm{kDa}$ major protein (lane 5, arrow), the absence of the 31.5- and 65-kDa minor proteins and reduced expression of the $90-\mathrm{kDa}$ protein. UG2 consisted of two isolates which had OMP profiles (Fig. 2, lanes 9 and 10) that were identical to the type 2.2.2 profile (Fig. 2, lane 8) of certain serotype A2 isolates (10).

UG3 isolates had OMP profiles (Fig. 1, lanes 9 through 12) similar to the type 3.2.2 profile (Fig. 1, lanes 7 and 8) of serotype A11 isolates (10). These profiles were characterized by the expression of a $40-\mathrm{kDa}$ major protein (Fig. 1, lane 7 , arrow) but also differed from the profiles of UG1 isolates in the mobilities of various high-molecular-mass minor proteins, as well as in the expression of minor $24-$ and $50-\mathrm{kDa}$ proteins (Fig. 1, lanes 6 and 7). Eight isolates had type 3.2.2 profiles. The profile of a single isolate, designated OMP type 3.2.3, differed from the other profiles in that the mobilities of the high-molecular-mass proteins and the expression of the 24and $50-\mathrm{kDa}$ proteins resembled the mobilities and expression

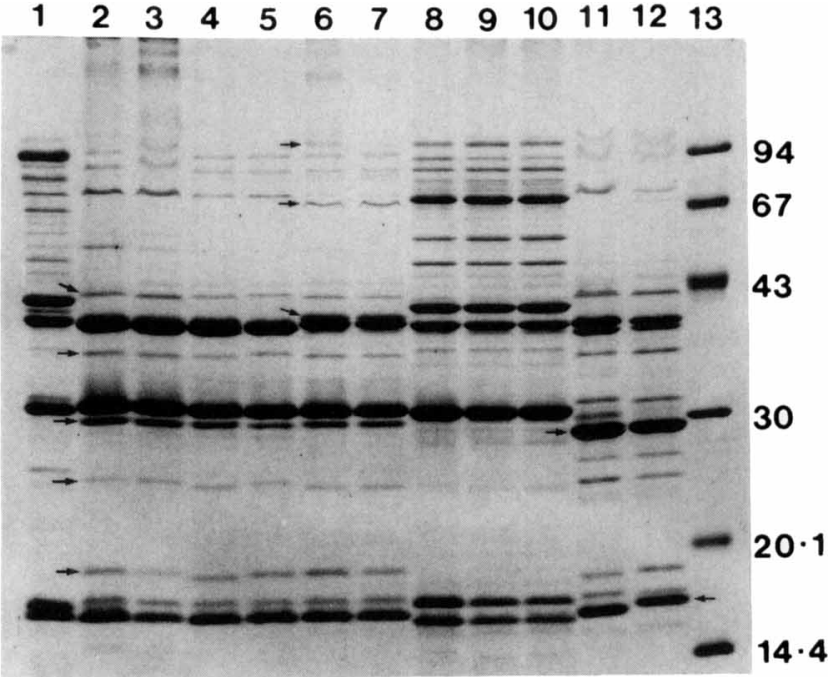

FIG. 2. OMP profiles of untypeable isolates of $P$. haemolytica showing OMP types 4.1.1 (lanes 2 through 5), 4.1 .2 (lanes 6 and 7), 2.2.2 (lanes 9 and 10), and 5.1.1 (lanes 11 and 12). Lane 1, OMP profile of a serotype A1 OMP type 1.1.1 isolate; lane 8, OMP profile of a serotype A2 OMP type 2.2 .2 isolate. Lane 13 contained molecular mass standards, whose positions (in kilodaltons) are indicated on the right.

found in the profiles of serotype A1 and UG1 isolates (Fig. 1, lane 12); however, this isolate was similar to the other UG3 isolates in its expression of a $40-\mathrm{kDa}$ major protein (lane 12, arrow). All of the profiles of members of this group resembled the type 1.2.1 profile of serotype A1 and UG1 isolates described above in that they lacked expression of 31.5- and 65$\mathrm{kDa}$ minor proteins (lane 3 , arrows) and contained a $30.5-\mathrm{kDa}$ major protein (lane 5, arrow). The significance of this is described below.

UG4 isolates had the characteristic OMP profiles shown in Fig. 2, lanes 2 through 7 . These profiles differed from the profiles of serotype A1 (UG1) and A2 (UG2) isolates (Fig. 2, lanes 1 and 8 ), as well as UG3 isolates, in the expression of both major and minor proteins. The main difference was in the expression of a single $38-\mathrm{kDa}$ major protein rather than two or three proteins in the $39-$ to $41-\mathrm{kDa}$ range, although this band may comprise two proteins that have similar molecular masses, as was evident in Fig. 2, lanes 6 and 7 (see below). In addition, the $31-\mathrm{kDa}$ major protein had a slightly higher molecular mass than the corresponding protein of serotype A1 isolates (Fig. 2, lanes 1 and 2). Other differences included the expression of 18.5-, 24.5-, 30-, 36-, and 42-kDa minor proteins (Fig. 2, lane 2, arrows) in UG4 isolates, as well as differences in the highmolecular-mass region. The UG4 isolates could be divided into two subgroups on the basis of differences in the mobilities of the major proteins in the 38.5- to 39.5-kDa range (Fig. 2, lane 6 , lower arrow) and in the expression of $67-$ and $100-\mathrm{kDa}$ proteins (Fig. 2, lane 6, upper arrows). The two profiles were designated OMP types 4.1 .1 (Fig. 2, lanes 2 through 5) and 4.1.2 (Fig. 2, lanes 6 and 7), although the profiles in Fig. 2, lanes 2 and 3, may represent a third type. Eight isolates had type 4.1.1 profiles, and three isolates had type 4.1.2 profiles.

UG5 comprised two isolates which had the OMP profile designated 5.1.1 (Fig. 2, lanes 11 and 12). This profile differed from the profiles of typeable isolates and other untypeable isolates of $P$. haemolytica in that it contained 28-, 38-, and $39-\mathrm{kDa}$ major proteins and various minor proteins with molecular masses ranging from 16 to $32 \mathrm{kDa}$. In particular, the 


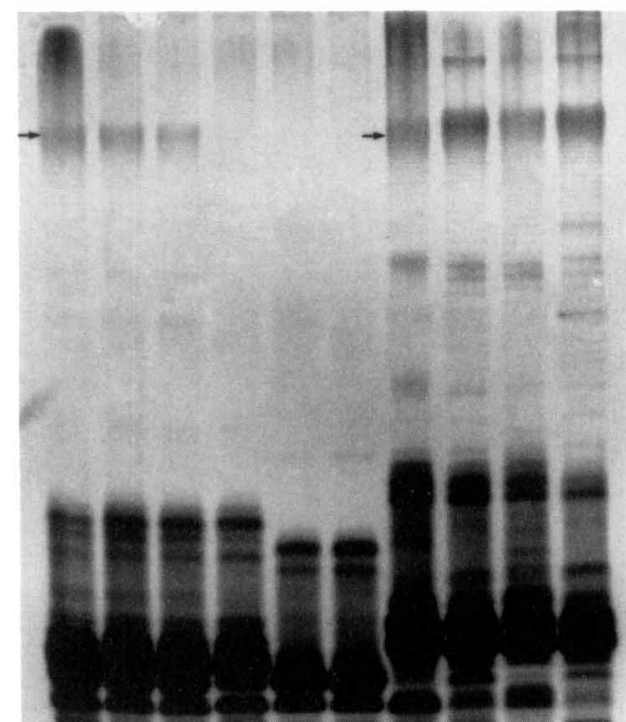

FIG. 3. LPS profiles of untypeable isolates of $P$. haemolytica showing LPS types $1 A$ (lanes 2 and 3), 3B (lanes 5 and 6 ), and $4 A / C$ (lanes 9 and 10). Lanes $1,4,7$, and 8, LPS types $1 \mathrm{~A}, 1 \mathrm{~B}, 4 \mathrm{~A}$, and $4 \mathrm{C}$ of serotype $\mathrm{A} 1, \mathrm{~A} 2, \mathrm{~A} 7$, and $\mathrm{A} 11$ isolates, respectively. The arrows indicate the ladder pattern of weakly staining $\mathrm{O}$-antigen side chains.

presence of a $28-\mathrm{kDa}$ major protein (Fig. 2, lane 11, arrow) rather than a $30-\mathrm{kDa}$ major protein and the presence of up to four minor proteins with molecular masses in the range 25 to $32 \mathrm{kDa}$ were distinguishing characteristics of this profile. Although there was variation between the two isolates in the mobilities of certain minor proteins (Fig. 2, lane 12, arrow), the profiles were given the same designation because only two isolates were available for comparison.

The LPS profiles of UG1 isolates were all smooth type 1A profiles (Fig. 3, lanes 2 and 3), whereas the profiles of UG2 isolates were all rough type $3 \mathrm{~B}$ profiles (Fig. 3 , lanes 5 and 6). The LPS profiles of the UG3 isolates were all similar to the smooth type 4A and 4C LPS profiles (Fig. 3, lanes 9 and 10) described for serotype A7 and A11 isolates (Fig. 3, lanes 7 and 8 ), respectively. However, at this time, the identities of the different LPS types in this group have not been confirmed immunologically (10), and the LPS types were all provisionally designated type $4 \mathrm{~A} / \mathrm{C}$. The LPS profiles of the remaining isolates could be divided into three discrete groups corresponding to their OMP profiles. Thus, the UG4 isolates that had type 4.1.1 OMP profiles had the variable LPS profiles shown in Fig. 4, lanes 2 through 5; the UG4 isolates that had type 4.1.2 OMP profiles had the identical profiles shown in Fig. 4, lanes 6 and 7; and the UG5 isolates that had type 5.1.1 OMP profiles had the identical LPS profiles shown in Fig. 4, lanes 8 and 9. Because there was no immunological confirmation of the identities of the LPS profiles in these groups, LPS types were not designated.

Phylogenetic analysis of the $P$. haemolytica complex by $16 \mathrm{~S}$ rRNA sequence comparison. The results of a comparison of the $16 \mathrm{~S}$ rRNA sequences of 28 isolates of $P$. haemolytica and $P$. trehalosi (Table 2) with the sequences of other members of the Pasteurellaceae indicated that the $P$. haemolytica isolates most closely resembled Bisgaard's taxa 15,18 , and 20, whereas the $P$. trehalosi isolates most closely resembled Actinobacillus capsulatus, Haemophilus paracuniculus, and Haemophilus parasuis. The similarity matrix for these comparisons is shown in Table

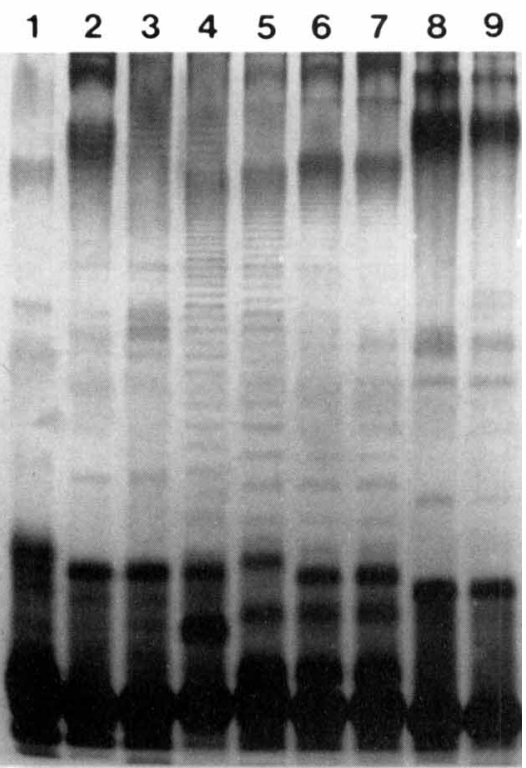

FIG. 4. LPS profiles of untypeable isolates of $P$. haemolytica showing the LPS profiles of OMP type 4.1.1 (lanes 2 through 5), 4.1.2 (lanes 6 and 7), and 5.1.1 (lanes 8 and 9) isolates. The type 1A LPS profile of a serotype A1 isolate is shown in lane 1.

4. A phylogenetic tree based on this similarity matrix is shown in Fig. 5. A total of 1,448 positions were used in the calculations, and positions with more than two missing nucleotides were not included in the analysis. The $P$. haemolytica isolates formed two distinct clusters, whereas the $P$. trehalosi isolates formed a third cluster on a separate branch (Fig. 5).

Serotype A1, A5 through A9, A12 through A14, and A16 isolates, together with untypeable isolate PH318 (UG1), formed a single group (the A1 group) whose members had identical 16S rRNA sequences. Two isolates each of serotypes $\mathrm{A} 1, \mathrm{~A} 2, \mathrm{~A} 6$, and A7 were examined because the isolates belonging to each pair could be differentiated on the basis of their OMP and/or LPS profiles (10). However, the results of a $16 \mathrm{~S}$ rRNA sequence comparison could not differentiate between the isolates of each of these pairs. Serotype A2 isolates, including type strain NCTC 9380, together with untypeable isolate PH690 (UG2), formed a second group (the A2 group), whose sequences differed from the sequences of members of the A1 group, as well as the sequences of the other isolates examined, in the substitution at base positions 646 and 743 of G by A. Serotype A11 isolate PH344 and untypeable isolate PH152 (UG3) together formed a group (0.7\% difference) which was most closely related to the main $P$. haemolytica group (Fig. 5). Isolates PH344 and PH152 and the members of the $\mathrm{A} 1$ and $\mathrm{A} 2$ groups differed by only 0.8 to $1.1 \%$, indicating that these taxa were closely related (Table 4). Untypeable isolate PH290 (UG3) was more distantly related to the main $P$. haemolytica group, whereas the isolate belonging to Bisgaard's taxon 18 was the most distantly related isolate in this cluster (Fig. 5). The remaining three untypeable isolates belonged to the second cluster, which also comprised Bisgaard's taxa 15 and 20 (Fig. 5). Isolates PH40 (UG4) and PH360 (UG4) were the most closely related isolates in this group, differing by only $0.6 \%$ (Table 4). Bisgaard's taxon 15 was the taxon that was next most closely related to isolates $\mathrm{PH} 40$ and $\mathrm{PH} 360$, followed by isolate PH704 (UG5) and Bisgaard's taxon 20 (Fig. 5).

The $P$. trehalosi isolates formed a distinct cluster which was most closely related to a group comprising $A$. capsulatus, $H$. 


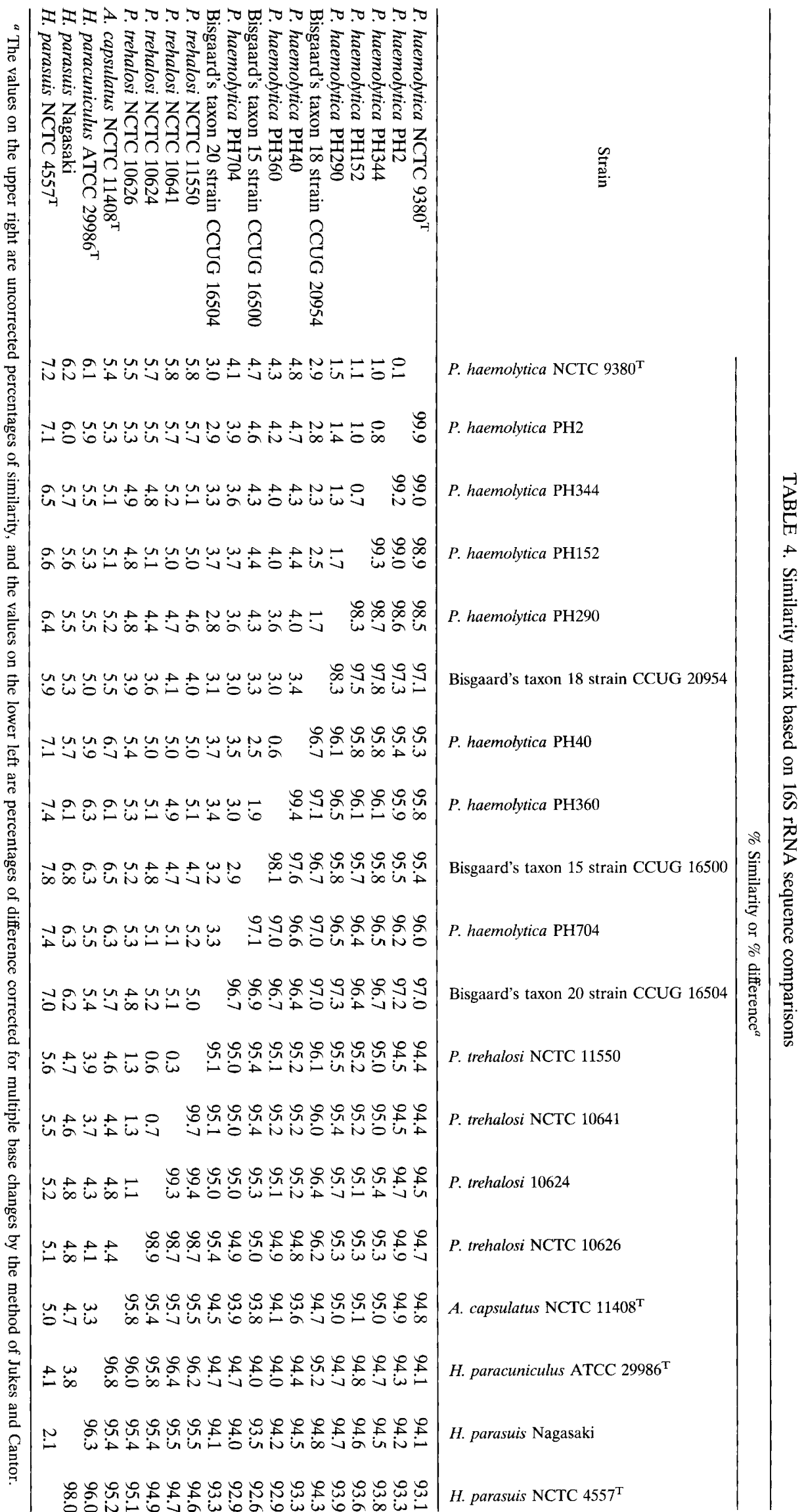




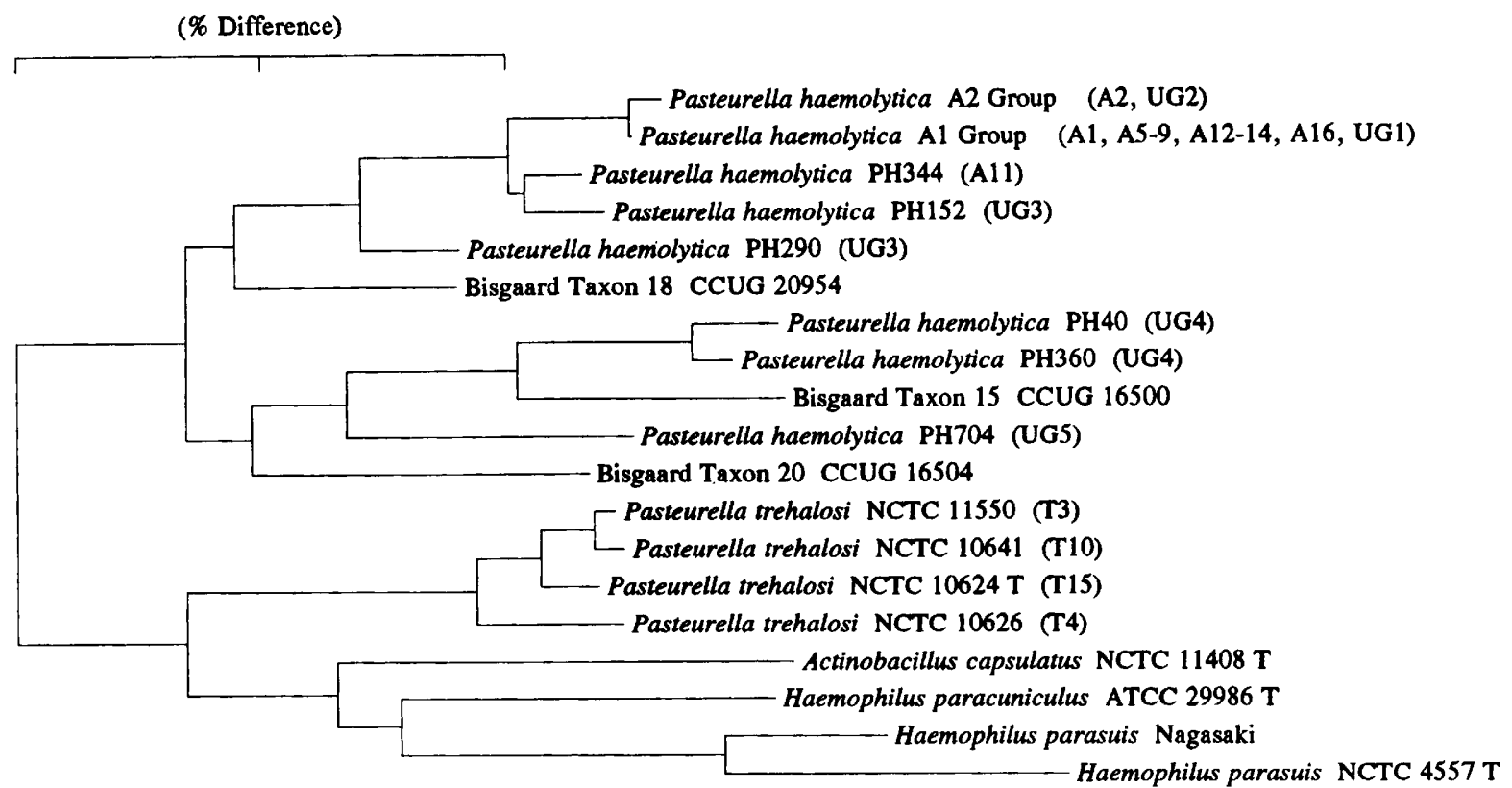

FIG. 5. Phylogenetic tree of the P. haemolytica complex and related taxa based on a $16 \mathrm{~S}$ rRNA sequence comparison. The A2 group consisted of isolates PH160, PH208, PH380, and PH690, and the A1 group consisted of isolates PH2, PH8, PH568, PH284, PH376, PH296, PH388, PH236, PH374, PH346, PH570, PH66, PH706, and $\mathrm{PH} 318$.

paracuniculus, and $H$. parasuis (Fig. 5). Isolates representing the four serotypes of $P$. trehalosi all had different $16 \mathrm{~S}$ rRNA sequences, unlike the $P$. haemolytica serotype A1, A5 through A9, A12 through A14, and A16 isolates, all of which had identical sequences. The sequence differences within $P$. trehalosi were as varied as the sequence differences observed between $P$. haemolytica serotypes A1, A2, and A11 and untypeable isolate PH152 (Table 4).

\section{DISCUSSION}

OMP and LPS profiles of untypeable isolates. The reproducibility of OMP and LPS analysis for assessing diversity within $P$. haemolytica and $P$. trehalosi has been described previously (10-12). The untypeable isolates could be divided into five major groups, UG1 through UG5, on the basis of their OMP and LPS profiles. The members of the two major groups, UG1 and UG2, were, except for having previously undescribed or no capsular polysaccharide structures, otherwise identical to corresponding serotype A1 and A2 isolates, respectively. In addition, the UG1 and UG2 isolates have the same host specificities as their serotype A1 and A2 counterparts. Like the serotype A1 isolates, the members of the two UG1 subgroups, represented by OMP types 1.1.1 and 1.2.1, were associated exclusively with cattle and sheep, respectively (Table 1). UG2 consisted of two ovine isolates whose OMP and LPS profiles were identical to the profiles of one of the two major groups of ovine serotype A2 isolates (i.e., OMP type 2.2.2 and LPS type 3B) (10). Whether these untypeable isolates have previously unidentified capsular structures needs to be resolved. UG1 included two isolates, PH318 (= UT3) and PH320 (=UT6), which previously were assigned to biogroup 1 of Bisgaard and Mutters (6); this biogroup also comprised serotype A1, A2, A5 through A9, and A12 through A14 isolates. A comparison of OMP and LPS profiles proved to be more discriminating than an analysis of biochemical characteristics since the former clearly differentiated among serotype A1, A2, and A7 isolates and also between isolates belonging to the same serotype (10), whereas the latter did not (6). However, it should be noted that isolate PH154 (= UT27), which could not be distinguished from other UG1 isolates on the basis of OMP and LPS profiles, was described by Bisgaard and Mutters (6) as a biogroup 9 isolate that was not able to produce ornithine decarboxylase. Biogroup 9 isolates could be differentiated from biogroup 1 isolates on the basis of $\mathrm{L}-(+)$-arabinose fermentation (6). Isolates phenotypically identical to PH154 (= UT27) were designated biogroup 10 by Bisgaard et al. (7).

UG3 probably corresponds to biogroup 5 of Bisgaard and Mutters since isolate PH150 (= UT18) was assigned to biogroup 5 by these authors (6). However, the similarity in OMP and LPS profiles between serotype A11 isolates, which were assigned to biogroup 3 by Bisgaard and Mutters (6), and UG3 isolates suggests that there is some overlap between biogroups 3 and 5. This group contained a single bovine isolate, but the eight other isolates belonging to the group were obtained from sheep (Table 1). This observation was consistent with the findings of Bisgaard and Mutters (6), since biogroup 5 consisted of four ovine strains, and suggests that UG3 is a sheep-specific taxon. The presence of a $30.5-\mathrm{kDa}$ major protein and the lack of expression of 31.5 - and $65-\mathrm{kDa}$ minor proteins were also characteristics of ovine-specific serotype A1 (UG1) and A2 (UG2) isolates and appear to be important features of ovine isolates (10). However, the pathogenic potential of isolates belonging to this group is uncertain because only three isolates were derived from the lungs of pneumonic sheep. Other isolates originated from diverse sites, such as an incidental oral lesion of the gingiva, the small intestine, semen, the stomach of an aborted fetus, and a mesenteric lymph node (Table 1).

UG4 probably corresponds to biogroup 6 of Bisgaard and Mutters since two isolates, PH40 (= UT35) and PH144 (=UT2), were assigned to this biogroup (6). All of the UG4 isolates except a single ovine isolate were recovered from cattle, as were the biogroup 6 isolates of Bisgaard and Mutters (6), suggesting that UG4 is a cattle-specific taxon. Although 
the majority of the isolates belonging to the group originated from the lungs of pneumonic cattle (as did the biogroup 6 isolates of Bisgaard and Mutters), two isolates were recovered from the placentae of aborted calves and a single isolate was obtained from a granuloma situated on the flank of an animal (Table 1). Two of these three isolates differed from the other strains in their OMP profiles, raising questions about the pathogenic potential of this subgroup of UG4.

The two UG5 isolates had OMP profiles that were distinct from the profiles of the other isolates examined and were also distinguished by the fact that they originated from an aborted placenta and a fetus (Table 1). The unique OMP profiles, together with the epidemiological data, suggest that these two isolates were less closely related to $P$. haemolytica than the other groups were and differed in their virulence potential.

16S rRNA sequences of members of the $P$. haemolytica complex. The $16 \mathrm{~S}$ rRNA sequence data confirmed the findings of a previous study (10) which indicated that serotypes A1, A2, and A11 represent three distinct groups within $P$. haemolytica. However, the sequence data could not be used to differentiate between serotype A7 isolates and other members of the A1 group, like the OMP-LPS analysis data (10). The sequence data also were consistent with the OMP and LPS data obtained in the present study which indicated that UG1 and UG2 correspond to serotypes $\mathrm{A} 1$ and $\mathrm{A} 2$, respectively. The OMP and LPS data obtained in a previous study (10), together with the sequence data obtained in the present study, suggest that biogroup 1 of Bisgaard and Mutters (6) is not a homogeneous group and that serotype A2 is a group which is distinct from the other serotypes. The differences in 16S rRNA sequences, together with the differences in OMP and LPS profiles (10), were consistent with placing serotype A11 in a separate biogroup, biogroup 3, as Bisgaard and Mutters did (6), and also confirmed the view of Frederiksen (18) that this serotype represents a third taxon.

The 16S rRNA sequence of isolate PH152, representing UG3, was only $0.7 \%$ different from the $16 \mathrm{~S}$ rRNA sequence of serotype A11 isolate PH344, suggesting that these organisms should be included in the same taxon. This finding supports the OMP and LPS data (see above), which suggests that there may be some overlap between biogroups 3 and 5 since Bisgaard and Mutters placed serotype A11 isolates in biogroup $3(6)$ and isolate PH152 may be affiliated with biogroup 5. However, the 16S rRNA sequence of untypeable isolate $\mathrm{PH} 290$, which also represents UG3, was $1.7 \%$ different from the $16 \mathrm{~S}$ rRNA sequence of isolate PH152 (Table 4). This difference was greater than the differences between isolate PH290 and the A1 and A2 groups ( 1.5 and $1.4 \%$, respectively) and indicated a heterogeneity in UG3 that was not apparent from the OMP and LPS data. Clearly, sequence data for additional isolates will be required to establish the full extent of variation within UG3 (biogroup 5) and also to determine the relationship of UG3 isolates to serotype A11 isolates (biogroup 3). However, PH152 was the only isolate in this group that originated from cattle, and the observed sequence difference between isolates PH152 and PH290 might be related to this fact. The sequence corresponding to Bisgaard's taxon 18 was the last member of this cluster (Fig. 5). However, since Bisgaard's taxon 18 consisted of a heterogeneous group of organisms, including at least four "biovars" (7), the single sequence available in the database is not necessarily representative of the taxon as a whole. Nevertheless, it was significant that all of the taxon 18 isolates were recovered from sheep, as were all but one of the UG3 isolates. The sequence data and epidemiological evidence suggest, therefore, that UG3 isolates and taxon 18 are closely related, although sequencing of additional isolates will be required to examine this suggestion in further detail.

UG4 isolates PH40 and PH360 were clearly closely related since their $16 \mathrm{~S}$ rRNA sequences differed by only $0.6 \%$. The level of similarity of the 16S rRNA sequences of these organisms was consistent with the minor differences observed in their OMP profiles. The sequence data (Fig. 5) suggested that UG4, which corresponded to biogroup 6 of Bisgaard and Mutters (6), not only was a species that was distinct from $P$. haemolytica, but also was less closely related to $P$. haemolytica than UG3 (biogroups 3 and 5), which belongs to a separate cluster. With the exception of a single isolate, all of the UG4 isolates originated from cattle, in contrast to the next most closely related group, taxon 15 , which comprised 13 strains isolated from pigs (5). On the basis of sequence data obtained from isolate PH704, UG5 represented a distinct taxon which was most closely related to Bisgaard's taxa 15 and 20 , the latter of which is represented by a single isolate obtained from the eye of a rabbit. This cluster of organisms therefore contained bacteria derived from a more diverse range of hosts, including cattle, pigs, and a rabbit, than the previous cluster, which originated from cattle and sheep.

$P$. trehalosi is now recognized as a separate species rather than biotype T of $P$. haemolytica (32). In a previous study (12) the OMP and LPS profiles of $P$. trehalosi were demonstrated to be significantly different from the profiles of $P$. haemolytica, an observation which is consistent with placing these organisms in different species. It was also shown that the level of variation in OMP and LPS profiles appeared to be less in P. trehalosi (12) than in $P$. haemolytica (10), suggesting that $P$. trehalosi is more homogeneous than $P$. haemolytica. However, the 16S rRNA sequence data suggested that the reverse is true since each of the four serotypes could be clearly differentiated from the others and the sequence of isolate NCTC 10626 (serotype T4) differed from the sequences of isolates NCTC 11550 (serotype T3) and NCTC 10641 (serotype T10) by $1.3 \%$ (Table 4). This was a greater difference than the difference between serotype A11 isolate $\mathrm{PH} 344$ and the $\mathrm{A} 1$ and $\mathrm{A} 2$ groups of $P$. haemolytica $(0.8$ to $1.0 \%)$. Although Bisgaard and Mutters (6) assigned serotype T4, T10, and T15 isolates to biogroup 2 and serotype $\mathrm{T} 3$ isolates to biogroup 4, our data suggest that there is greater variation among serotype $\mathrm{T} 4, \mathrm{~T} 10$, and $\mathrm{T} 15$ isolates than previously thought. Variation within $P$. trehalosi clearly needs to be investigated further. The results obtained with $P$. trehalosi support the conclusion of Clayton et al. (8) that sequences obtained from single operons from single isolates may inadequately represent their taxa in phylogenetic studies and biodiversity estimates.

In summary, the usefulness of OMP and LPS analysis as a taxonomic aid in the study of the relationships of isolates belonging to the $P$. haemolytica complex was clearly demonstrated in the present study since conclusions arrived at on the basis of OMP and LPS data were supported by 16S rRNA sequence data. Analysis of OMP and LPS profiles was found to be particularly suitable for gaining an overall impression of the taxonomic relationships of a relatively large number of isolates, although the technique cannot demonstrate phylogenetic relationships, as $16 \mathrm{~S}$ rRNA sequence comparisons can. The resolving power of the OMP and LPS analysis was, in most cases, greater than the resolving power of 16S rRNA sequence comparisons, as demonstrated by the results of the analysis of typeable $P$. haemolytica isolates (10). However, in the case of isolates belonging to UG3, as well as $P$. trehalosi isolates, the reverse was true; $16 \mathrm{~S}$ rRNA sequence comparisons seemed to have greater resolving power than OMP and LPS analysis. The ability to differentiate between untypeable isolates belonging 
to the $P$. haemolytica complex and between isolates of the same serotype demonstrated the usefulness of OMP and LPS analysis in epidemiological studies. Both techniques revealed that UG1 and $\mathrm{UG} 2$ correspond to serotypes $\mathrm{A} 1$ and $\mathrm{A} 2$, respectively; that UG3 is closely related to serotype A11; and that UG4 and UG5 are more distantly related to $P$. haemolytica and represent two different species.

\section{ACKNOWLEDGMENTS}

This investigation was supported by Wellcome Taxonomy Fellowship 038464/Z/93/Z/REH/MW to R. L. Davies.

This study was made possible by the assistance and cooperation of various individuals at the following Veterinary Investigation Centres: Aberdeen, Auchincruvie, Dumfries, Edinburgh, and St. Boswells in Scotland, Bury St. Edmunds, Langford, Newcastle, Penrith, Shrewsbury, and Sutton Bonington in England, and Aberystwyth and Carmarthen in Wales. We are particularly grateful to David Dyson (Penrith) and Chris Low (Edinburgh) for their interest, advice, and help and to Nia Tzellas (Boston, Mass.) for guidance with the sequencing.

\section{REFERENCES}

1. Aarsleff, B., E. L. Biberstein, B. J. Shreeve, and D. A. Thompson. 1970. A study of untypable strains of Pasteurella haemolytica. J. Comp. Pathol. 80: 493-498.

2. Adlam, C. 1989. The structure, function and properties of cellular and extracellular components of Pasteurella haemolytica, p. 75-92. In C. Adlam and M. J. Rutter (ed.), Pasteurella and pasteurellosis. Academic Press, London.

3. Biberstein, E. L. 1978. Biotyping and serotyping of Pasteurella haemolytica. Methods Microbiol. 10:253-269.

4. Biberstein, E. L., and C. K. Francis. 1968. Nucleic acid homologies between the $\mathrm{A}$ and $\mathrm{T}$ types of Pasteurella haemolytica. J. Med. Microbiol. 1:105-108.

5. Bisgaard, M. 1984. Comparative investigations of Pasteurella haemolytica sensu stricto and so-called $P$. haemolytica isolated from different pathological lesions in pigs. Acta Pathol. Microbiol. Immunol. Scand. Sect. B 92:201-207.

6. Bisgaard, M., and R. Mutters. 1986. Re-investigations of selected bovine and ovine strains previously classified as Pasteurella haemolytica and description of some new taxa within the Pasteurella haemolytica complex. Acta Pathol. Microbiol. Immunol. Scand. Sect. B 94:185-193.

7. Bisgaard, M., J. E. Phillips, and W. Mannheim. 1986. Characterization and identification of bovine and ovine Pasteurellaceae isolated from the oral cavity and rumen of apparently normal cattle and sheep. Acta Pathol. Microbiol. Immunol. Scand. Sect. B 94:9-17.

8. Clayton, R. A., G. Sutton, P. S. Hinkle, C. Bult, and C. Fields. 1995. Intraspecific variation in small-subunit rRNA sequences in GenBank: why single sequences may not adequately represent prokaryotic taxa. Int. J. Syst. Bacteriol. 45:595-599.

9. Davies, R. L., O. Ali, R. Parton, J. G. Coote, H. A. Gibbs, and J. H. Freer. 1991. Optimal conditions for the analysis of Pasteurella haemolytica lipopolysaccharide by sodium dodecyl sulphate-polyacrylamide gel electrophoresis. FEMS Microbiol. Lett. 90:23-28.

10. Davies, R. L., and W. Donachie. 1996. Intra-specific diversity and host specificity within Pasteurella haemolytica based on variation of capsular polysaccharide, lipopolysaccharide and outer membrane proteins. Microbiology, 142:1895-1907.

11. Davies, R. L., R. Parton, J. G. Coote, H. A. Gibbs, and J. H. Freer. 1992 Outer membrane protein and lipopolysaccharide variation in Pasteurella haemolytica A1 under different growth conditions. J. Gen. Microbiol. 138: 909-922.

12. Davies, R. L., and M. Quirie. 1996. Intra-specific diversity within Pasteurella trehalosi based on variation of capsular polysaccharide, lipopolysaccharide and outer membrane proteins. Microbiology 142:551-560.

13. Dewhirst, F. E., B. J. Paster, I. Olsen, and G. J. Fraser. 1992. Phylogeny of 54 representative strains of species in the family Pasteurellaceae as determined by comparison of 16S rRNA sequences. J. Bacteriol. 174:2002-2013.

14. Donachie, W., J. Fraser, M. Quirie, and N. J. L. Gilmour. 1984. Studies on strains of Pasteurella haemolytica not typable by the indirect haemagglutination test. Res. Vet. Sci. 37:188-193.

15. Filip, C., G. Fletcher, J. L. Wulff, and C. F. Earhart. 1973. Solubilization of the cytoplasmic membrane of Escherichia coli by the ionic detergent sodium lauryl sarcosinate. J. Bacteriol. 115:717-722.

16. Frank, G. H. 1989. Pasteurellosis of cattle, p. 197-222. In C. Adlam and M. J. Rutter (ed.), Pasteurella and pasteurellosis. Academic Press, London.

17. Fraser, J., S. Laird, and N. J. L. Gilmour. 1982. A new serotype (biotype T) of Pasteurella haemolytica. Res. Vet. Sci. 32:127-128.

18. Frederiksen, W. 1973. Pasteurella taxonomy and nomenclature. Contrib. Microbiol. Immunol. 2:170-176.

19. Gilmour, N. J. L., and J. S. Gilmour. 1989. Pasteurellosis of sheep, p. 223-261. In C. Adlam and M. J. Rutter (ed.), Pasteurella and pasteurellosis. Academic Press, London.

20. Hitchcock, P. J., and T. M. Brown. 1983. Morphological heterogeneity among Salmonella lipopolysaccharide chemotypes in silver-stained polyacrylamide gels. J. Bacteriol. 154:269-277.

21. Jukes, T. H., and C. R. Cantor. 1969. Evolution of protein molecules, p. 21-132. In H. N. Munro (ed.), Mammalian protein metabolism, vol. 3. Academic Press, Inc., New York.

22. Kusukawa, N., T. Uemori, K. Asada, and I. Kato. 1990. Rapid and reliable protocol for direct sequencing of material amplified by the polymerase chain reaction. BioTechniques 9:66-72.

23. Laemmli, U. K. 1970. Cleavage of structural proteins during the assembly of the head of bacteriophage T4. Nature (London) 227:680-685.

24. Markwell, M. A. K., S. M. Haas, L. L. Bieber, and N. E. Tolbert. 1978. A modification of the Lowry procedure to simplify protein determination in membrane and lipoprotein samples. Anal. Biochem. 87:206-210.

25. Mutters, R., M. Bisgaard, and S. Pohl. 1986. Taxonomic relationship of selected biogroups of Pasteurella haemolytica as revealed by DNA:DNA hybridizations. Acta Pathol. Microbiol. Immunol. Scand. Sect. B 94:195-202.

26. Mutters, R., P. Ihm, S. Pohl, W. Frederiksen, and W. Mannheim. 1985. Reclassification of the genus Pasteurella Trevisan 1887 on the basis of deoxyribonucleic acid homology, with proposals for the new species Pasteurella dagmatis, Pasteurella canis, Pasteurella stomatis, Pasteurella anatis, and Pasteurella langaa. Int. J. Syst. Bacteriol. 35:309-322.

27. Mutters, R., W. Mannheim, and M. Bisgaard. 1989. Taxonomy of the group, p. 3-34. In C. Adlam and M. J. Rutter (ed.), Pasteurella and pasteurellosis. Academic Press, London.

28. Paster, B. J., and F. E. Dewhirst. 1988. Phylogeny of campylobacters, wolinellas, Bacteroides gracilis, and Bacteroides ureolyticus by $16 \mathrm{~S}$ ribosomal ribonucleic acid sequencing. Int. J. Syst. Bacteriol. 38:56-62.

29. Quirie, M., W. Donachie, and N. J. L. Gilmour. 1986. Serotypes of Pasteurella haemolytica from cattle. Vet. Rec. 119:93-94.

30. Saitou, N., and M. Nei. 1987. The neighbor-joining method: a new method for reconstructing phylogenetic trees. Mol. Biol. Evol. 4:406-425.

31. Sneath, P. H. A., and M. Stevens. 1985. A numerical taxonomic study of Actinobacillus, Pasteurella and Yersinia. J. Gen. Microbiol. 131:2711-2738.

32. Sneath, P. H. A., and M. Stevens. 1990. Actinobacillus rossii sp. nov., Actinobacillus seminis sp. nov., nom. rev., Pasteurella bettii sp. nov., Pasteurella lymphangitidis sp. nov., Pasteurella mairi sp. nov., and Pasteurella trehalosi sp. nov. Int. J. Syst. Bacteriol. 40:148-153.

33. Studier, J., and K. Keppler. 1988. A note on the neighbor-joining algorithm of Saitou and Nei. Mol. Biol. Evol. 5:729-731.

34. Tsai, C. M., and C. E. Frasch. 1982. A sensitive silver stain for detecting lipopolysaccharide in polyacrylamide gels. Anal. Biochem. 119:115-119.

35. Younan, M., and I. Fodor. 1995. Characterization of a new Pasteurella haemolytica serotype (A17). Res. Vet. Sci. 58:98. 